Original

\title{
Complicaciones del tratamiento quirúrgico tras radioterapia y quimioterapia neoadyuvantes en el cáncer no microcítico de pulmón. Análisis de una serie de 16 pacientes
}

\author{
D. Gómez de Antonio ${ }^{1}$, C. de la Fuente ${ }^{2}$, A. Hurtado ${ }^{3}$, I. Zapata ${ }^{2}$, P. Gámez ${ }^{1}$, M. Córdoba ${ }^{1}$, A. Varela ${ }^{1}$
}

\section{Resumen}

Propósito: Comparar la morbimortalidad postoperatoria de pacientes con cáncer de células no pequeñas de pulmón tratados con dosis completas de radioterapia de inducción ( $>59$ Gy) y con dosis estándar de radioterapia ( $\leq 5 \mathrm{~Gy}$ ).

Material y métodos: Revisamos los pacientes con CNMP que desde diciembre del año 2000 recibieron tratamiento quirúrgico con intención curativa tras quimioradioterapia \pm quimioterapia de inducción en nuestro centro $(n=16$.). Se recogen los datos de complicaciones postoperatorias, tiempo y localización de las recidivas, metástasis y supervivencia de los pacientes.

Resultados: No se produjo ningún caso de mortalidad perioperatoria. Las diferencias en la incidencia de complicaciones postoperatorias en ambos grupos no es estadísticamente significativa (p<0,197). La supervivencia actuarial a los 2 años es del 66,6\%.

Conclusión: En nuestro medio parece seguro el tratamiento quirúrgico tras dosis plenas de radioterapia en el CNMP.

Palabras clave:

Tratamiento de inducción. Cirugía. Carcinoma no microcítico de pulmón localmente avanzado. Morbimortalidad.

Oncología, 2006; 29 (2):65-72

\footnotetext{
${ }^{1}$ Servicio de Cirugía Torácica

${ }^{2}$ Servicio de Oncología Radioterápica

${ }^{3}$ Servicio de Oncología Médica

Hospital Universitario Puerta de Hierro

Madrid (España)
}

Recibido: 29.11 .05

Aceptado: 08.02.06 


\section{Summary}

Purpose: To compare the postoperative morbimortality of patients with non-small cell lung cancer (NSCLC) patients treated employing a full dose of induction radiotherapy ( $>59 \mathrm{~Gy}$ ) with those receiving a standard dose of radiotherapy ( $\leq 45 \mathrm{~Gy}$ ).

Material and methods: A retrospective analysis of patients with NSCLC treated by surgery after chemo-radiotherapy \pm chemotherapy in our institution since December $2000 \quad(n=16)$ is made. Data about survival, postoperative morbidity, and time and site of recurrence or metastases are considered.

Results: There was no perioperative mortality. No statistical differences were found in postoperative morbidity when comparing both groups of radiation $(\mathrm{p}<0.197)$. The two years actuarial survival rate was $66.6 \%$.

Conclusion: In our experience, it seems reasonable to offer surgery to patients with NSCLC even when they have received a full dose induction radiotherapy.

Key words: Induction therapy. Surgery. Non-small cell lung cancer. Morbimortality.

\section{Introducción}

El cáncer de pulmón es la principal causa de muerte de etiología tumoral. El carcinoma no microcítico de pulmón (CNMP) representa las tres cuartas partes de los cánceres de este órgano ${ }^{1}$. En el momento del diagnóstico, cerca de $50 \%$ de los pacientes tienen extensión extratorácica, y un $10-15 \%$ presentan enfermedad localmente avanzada en pared torácica, diafragma o mediastino. De éstos, la mayoría no son candidatos a tratamiento quirúrgico de entrada, y pueden ser incluidos en esquemas de tratamiento con la asociación de radioterapia torácica y quimioterapia secuencial o concomitante en un intento de hacer resecables tumores que al diagnóstico no lo eran.

Después del tratamiento de inducción se evalúa la respuesta tumoral y ganglionar mediante pruebas de imagen (tomografía axial computerizada (TAC), tomografía por emisión de positrones (PET), resonancia magnética nuclear (RMN)) y/o pruebas invasivas (fibrobroncoscopia/esofagoscopia con punción aspirativa, mediastinoscopia, toracoscopia) y aquellos con respuesta parcial o completa se pueden rescatar para cirugía, alcanzando de esta manera una supervivencia a los cinco años entre $5-25 \%{ }^{2,3}$.

Diversos estudios han sugerido un incremento notable de la morbimortalidad postoperatoria de los pacientes sometidos a tratamiento de inducción, especialmente si han recibido radioterapia a dosis máximas, por encima de $59 \mathrm{~Gy}^{4-10}$. Sin embargo, la experiencia de otros autores no ha confirmado este hecho ${ }^{11-17}$. El objetivo de nuestro trabajo es analizar si el empleo de dosis plenas de radioterapia se asocia a una mayor incidencia de complicaciones postoperatorias en nuestro medio, así como describir la supervivencia de los pacientes con CNMP tratados con quimioradioterapia \pm quimioterapia y cirugía.

\section{Material y métodos}

Revisamos retrospectivamente todos los pacientes con CNMP que entre diciembre del año 2000 y Noviembre del 2004 recibieron tratamiento quirúrgico con intención curativa tras quimioradioterapia \pm quimioterapia de inducción en el Hospital Universitario Puerta de Hierro de Madrid.

Incluimos en nuestro estudio aquellos pacientes con cáncer de pulmón con diagnóstico anatomopatológico de no microcítico que cumplen alguno de los siguientes criterios: estadio clínico (c)T3N $>1$, cT4 sin derrame pleural, cT3 o cT4 Pancoast \pm síntomas, $\mathrm{cN}>2$ o cM1 única en cerebro. 
TABLA I

Estadios clínicos

\begin{tabular}{|lcc|}
\hline Estadios & $n=16(\%)$ & Pancoast \\
\hline IIBc & $4(25 \%)$ & 3 \\
IIIAc & $4(25 \%)$ & 2 \\
IIIBc & $8(50 \%)$ & 2 \\
\hline
\end{tabular}

$\mathrm{n}=$ número de pacientes

Todos los pacientes completaron su estudio mediante escáner toracoabdominal y en algún caso PET. El diagnóstico anatomopatológico se obtuvo mediante fibrobroncoscopia con toma de biopsias en 10 casos $(62,5 \%)$ y por punción aspiración con aguja fina (PAAF) transtorácica en 6 casos (37,5\%). Se tomaron muestras de las adenopatías sospechosas $(>1 \mathrm{~cm}$ en TAC) mediante mediastinoscopia en todos los pacientes, fibrobroncoscopia en $6(37,5 \%)$ y toracoscopia en $3(16,25 \%)$. Cuando la clínica así lo sugería, se realizó Gammagrafía ósea y RMN cerebral para detectar enfermedad diseminada. Asímismo, en algún caso de tumor de Pancoast, se rea- lizó RMN, arteriografía y/o venografía para descartar afectación vascular. Por último se hicieron pruebas de función respiratoria a todos los pacientes candidatos a tratamiento quirúrgico. Se desestimaron para cirugía aquellos pacientes con un flujo espiratorio máximo en el primer segundo predicho postoperatorio (FEV1 pp) menor de $800 \mathrm{ml}$, historia de angina inestable o infarto en los 3 meses previos o clase funcional de la NYHA III-IV.

Finalmente incluimos 16 pacientes en el estudio, 4 mujeres $(25 \%)$ y 12 hombres $(75 \%)$, con una media de edad de 57,43 años (rango 42-77a). Las estirpes histológicas de los tumores eran $5(31,25 \%)$ epidermoides, 4 (25\%) adenocarcinomas, 1 (6,25\%) indiferenciado de células grandes y $6(37,5 \%)$ carcinomas no microcíticos indeterminados. En cuanto a los estadios clínicos tumorales pre-neoadyuvancia (c) (Tabla I), $4(25 \%)$ eran cT3N0M0, 2 (12,5\%) cT2N2M0, 2 $(12,5 \%)$ cT3N2M0, $3(18,75 \%)$ cT4N0M0, 3 $(18,75 \%)$ cT4N2M0, $1(6,25 \%)$ cT2N3M0, y 1 $(6,25 \%)$ cT4N3M0. De todos ellos había 7 pacientes con tumores de Pancoast, 3 cT3NOM0, 1 cT4NOM0, 2 cT3N2M0 y 1 cT4N2M0.

TABLA II

Grupos de dosis y comparación de variables

\begin{tabular}{|c|c|c|c|}
\hline & $\leq 50 G y$ & $>59 G y$ & $P$ \\
\hline $\mathrm{N}$ & 11 & 5 & \\
\hline Dosis (Gy) & $X=45(\mathrm{DS} 1,15)$ & $X=61,2(D S: 2,17)$ & \\
\hline Edad (años) & $\mathrm{X}=56,36(\mathrm{DS} 9,93)$ & $X=59,80($ DS 11,50$)$ & $0,55 \mathrm{NS}$ \\
\hline FEV1 $(\%)$ & $X=90,91(D S 18,81)$ & $X=80,20(\mathrm{DS} 15,79)$ & $0,28 \mathrm{NS}$ \\
\hline Género & H $9(81,8 \%)$ M $2(18,2 \%)$ & H $3(60 \%)$ M $2(40 \%)$ & $0,75 \mathrm{NS}$ \\
\hline \multirow[t]{2}{*}{ Antecedentes personales } & CV $3(17,3 \%)$ EPOC $1(9,1 \%)$ & & \\
\hline & NO $7(63,6 \%)$ & EPOC $1(20 \%)$ NO $4(80 \%)$ & $0,40 \mathrm{NS}$ \\
\hline \multirow[t]{2}{*}{ Histología } & Adenoca $3(27,3 \%)$ Epid $3(27,3 \%)$ & Adenoca 1 (20\%) Epid 2 (40\%) & \\
\hline & CG $1(9,1 \%)$ Indet $4(36,4 \%)$ & Epid.+CG 1 (20\%) Indet $1(20 \%)$ & $0,52 \mathrm{NS}$ \\
\hline Pancoast & $5(45,4 \%)$ & $2(40 \%)$ & $0,64 \mathrm{NS}$ \\
\hline Quimioterapia & $6(54,5 \%)$ & $5(100 \%)$ & $0,19 \mathrm{NS}$ \\
\hline Neumonectomía & $1(9,09 \%)$ & $3(60 \%)$ & $0,63 \mathrm{NS}$ \\
\hline $\mathrm{E}(\mathrm{c})$ & IIB $4(36,4 \%)$ IIIA $3(27,3 \%)$ IIIB $4(36,4 \%)$ & IIIA1(20\%) IIIB4(80\%) & $0,20 \mathrm{NS}$ \\
\hline \multirow[t]{2}{*}{ E post } & IB1 $(9,1 \%)$ IIB5(45,4\%) IIIA1 $(9,1 \%)$ & & \\
\hline & IIIB $4(36,4 \%)$ & NO2(40\%) IIIA1(20\%) IIIB2(40\%) & $0,11 \mathrm{NS}$ \\
\hline \multirow[t]{2}{*}{$\mathrm{E}(\mathrm{p})$} & NO $5(45,4 \%)$ IIB $1(9,1 \%)$ & NO $2(40 \%)$ IIB $1(20 \%)$ & \\
\hline & IIIA $3(27,3 \%)$ IIIB $2(18,2 \%)$ & IIIA $1(20 \%)$ IIIB $1(20 \%)$ & $0,73 \mathrm{NS}$ \\
\hline
\end{tabular}

$\mathrm{N}$ : número de pacientes.

Gy: Grays

X:media DS: desviación estándar.

H: hombre. M: mujer

FEV1: volumen espirado máximo en el primer segundo.

$\mathrm{CV}$ : cardiovasculares (Hipertensión arterial, arritmias, infarto previo).
EPOC: enfermedad pulmonar obstructiva crónica.

Adenoca: adenocarcinoma. Epid: epidermoide. Indet: indeterminado. CG: células grandes

E(c): estadio clínico. Epost: estadio tras tratamiento de inducción. E(p): estadio patológico.

NO: no evidencia de tumor. NS: no significativo. 
El estadio patológico (p) se establece de acuerdo con el sistema internacional de estadificación del cáncer de pulmón ${ }^{18}$.

El tratamiento quimioterápico neoadyuvante se empleó en 11 pacientes $(68,75 \%), 4$ (36,6\%) recibieron una combinación de cisplatino y gemcitabina (uno de ellos además recibió taxotere), 6 (54,5\%) cisplatino más vinorelbina y 1 (9\%) una combinación de carboplatino y taxol.

La radioquimioterapia se planificó tridimensionalmente a partir de las imágenes de escáner previas al tratamiento en todos los casos, y a todos los pacientes se les administró de manera concurrente ciclos de taxol $\left(60 \mathrm{mg} / \mathrm{m}^{2}\right.$ semanalmente $3 \mathrm{~h}$ antes de la radioterapia) excepto en dos casos, a uno se le dio vinorelbina $\left(15 \mathrm{mg} / \mathrm{m}^{2}\right.$ semanalmente 30 minutos antes de la radioterapia), y otro paciente desarrolló una polineuropatía relacionada con el taxol que obligó a cambiarlo por topotecán (0.5 miligramos/ $\mathrm{m}^{2}$ x 3 días cada 21 días). Se retrasaba el ciclo siguiente hasta que la cifra de neutrófilos fuera superior a $1.500 / \mathrm{mm}^{3}$ y la de plaquetas mayor de $100.000 / \mathrm{mm}^{3}$. Se reducían al $20 \%$ las dosis del quimioterápico si la cifra de neutrófilos estaba por debajo de $500 / \mathrm{mm}^{3}$ y se suspendía si aparecía neuropatía severa. La radioterapia se aplicó concomitantemente de manera fraccionada (1.8 Gy/día) sobre el tumor, y las áreas linfáticas clínicamente sospechosas. 5 pacientes $(31,25 \%)$ recibieron una dosis acumulada mayor de 59Gy (media 61,2Gy; desviación estándar (DS) 2,17) y el resto, 11 pacientes $(68,75 \%)$, recibieron una dosis acumulada entre 40 y 50Gy (media 45Gy; DS 1,15) (Tabla II).

Posteriormente se reestadificó cada paciente mediante escáner y, cuando fue necesario, PET y/o RMN. Se decidió tratamiento quirúrgico cuando se observaba respuesta parcial o completa tumoral y ganglionar. Se realizó la intervención quirúrgica con un intervalo de tiempo desde la última dosis de radioterapia de alrededor de 57 días (media 56,71d; rango 10-135d).

La cirugía se llevó a cabo con intención curativa en todos los casos. Se llevaron a cabo $11(68,75 \%)$ lobectomías, $1(6,25 \%)$ bilobectomía y 4 (25\%) neumonectomías izquierdas. Hubo 7 lobectomías que requirieron resección en bloque de al menos 2 costillas, una de ellas precisó además resección de la arteria subclavia que se reconstruyó con una prótesis de Gore-Tex ${ }^{\circledR}$ y otras 3 requirieron resección
TABLA III

\begin{tabular}{|lc|}
\multicolumn{1}{c}{} & Resecciones ampliadas \\
\hline \multicolumn{1}{|c|}{$N$} \\
\hline Pericardio & $3(18,75 \%)$ \\
Arteria subclavia & $1(6,25 \%)$ \\
Plexo braquial & $3(18,75 \%)$ \\
Aurícula izquierda & $1(6,25 \%)$ \\
Pared torácica & $7(43,5 \%)$ \\
\hline
\end{tabular}

de algún tronco del plexo braquial. Tres de las 4 neumonectomías fueron intrapericárdicas, y en un caso hubo que resecar una porción de la aurícula izquierda (Tabla III). En todas las neumonectomías se asoció plastia del muñón bronquial (una con pericardio, otras 2 con grasa mediastínica y otra con pleura parietal) y en 7 de los 12 casos restantes también se aplicó algún procedimiento de cobertura del bronquio seccionado (en un caso con pericardio, 2 casos con grasa mediastínica, otros 2 casos con pleura parietal, un caso con pleura mediastínica y el último con músculo serrato). En todos los casos la sección del bronquio se realizó con bisturí frío tras el cierre de la luz bronquial con máquina grapadora, y siempre se dieron puntos sueltos con sutura 4-0 monofilamento reabsorbible en el borde libre bronquial. El manejo postoperatorio de los pacientes sometidos a resección pulmonar se basa en un manejo estricto del balance hídrico, analgesia adecuada, fisioterapia respiratoria y movilización precoz desde las primeras horas del postoperatorio.

Se define resección completa como la ausencia de enfermedad en los márgenes quirúrgicos de la pieza resecada tras su análisis anatomopatológico definitivo. En todos los casos se obtuvieron márgenes quirúrgicos libres.

Finalmente se recogen las complicaciones postoperatorias (neumonía, fístula del muñón bronquial, empiema, distrés respiratorio, hemotórax, infarto agudo de miocardio, arritmias, parálisis frénica, síndrome de Horner, insuficiencia renal, trombosis venosa profunda y tromboembolismo pulmonar, fuga aérea persistente, infección urinaria, disfonía, atelectasias e infección de la herida quirúrgica), así como las fechas y localización de las recidivas y los datos de supervivencia de los pacientes

Los datos se recogen en una base de datos Microsoft Excel, se comparan las diferentes variables ana- 
lizadas entre los dos grupos de dosis de radioterapia (menor o igual a 45 Gy y mayor de 59 Gy) mediante la prueba de Chi cuadrado para variables cualitativas y $\mathrm{T}$ de Student para variables cuantitativas para averiguar la homogeneidad de ambos grupos. El análisis de la supervivencia se realiza por el método de Kaplan-Meier.

\section{Resultados}

No hubo ningún caso de mortalidad operatoria ni postoperatoria.

En 7 pacientes (43\%) se evidenció alguna complicación postquirúrgica. En el grupo de pacientes que recibieron dosis mayores de 59 Gy hubo un caso de disfonía por sección del nervio recurrente laríngeo, mientras que en el grupo que recibió dosis menores o iguales a 50 Gy se evidenciaron las siguientes complicaciones postquirúrgicas: un síndrome de Horner por lesión del ganglio estrellado, un caso de disfonía por sección del nervio recurrente laríngeo, una neumonía en la primera semana postoperatoria, un hemotórax postquirúrgico que se manejó de manera conservadora, un caso de empiema que precisó tratamiento antibiótico y drenaje con tubo de tórax y un infarto agudo de miocardio. Las diferencias en la incidencia de complicaciones postoperatorias en ambos grupos no resultó ser estadísticamente significativa $(\mathrm{p}<0,197)$ (Figura 1).

La comparación en la distribución de las diferentes variables analizadas en ambos grupos (edad, se-

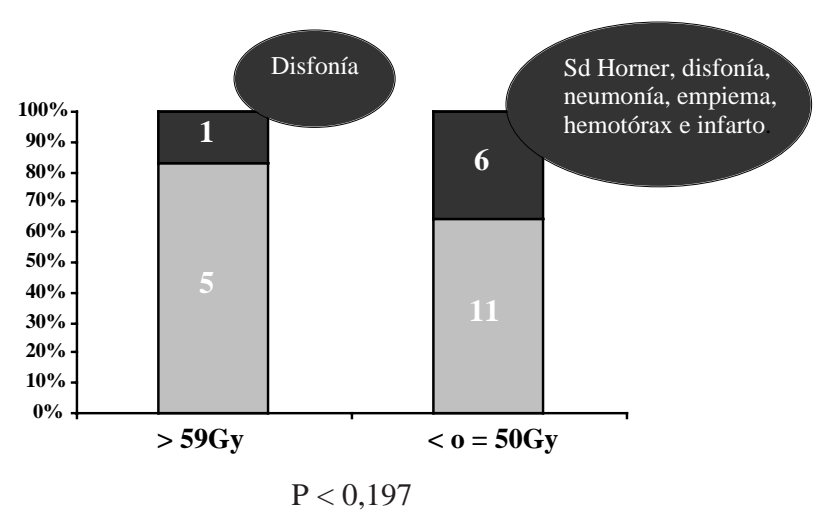

Figura 1. Complicaciones postquirúrgicas.

xo, antecedentes personales, función pulmonar, quimioterapia previa, tipo histológico, tipo de cirugía y estadios tumorales) no resultó estadísticamente significativa (Tabla II), aunque para el tipo de cirugía se observa una tendencia en cuanto al número de neumonectomías a favor del grupo de pacientes que recibió más dosis de radioterapia.

En cuanto a la respuesta al tratamiento neoadyuvante (Tabla IV), 11 pacientes $(68,75 \%)$ respondieron al tratamiento obteniéndose un estadio patológico (p) inferior al clínico. Ningún paciente sufrió progresión de su enfermedad durante el tratamiento preoperatorio, y 5 pacientes $(31,25 \%)$ permanecieron en el mismo estadio. En 7 pacientes $(46,6 \%)$ no hubo evidencia anatomopatológica de tumor viable. Hubo 8 pacientes con enfermedad $\mathrm{cN}>1$, de los cuales $5(62,5 \%)$ resultaron ser pN0 y $1(12,5 \%)$ pN1.

En cuanto a la serie global, la media de supervivencia fue de 18,3 meses, mientras que la media de

TABLA IV

Respuesta al tratamiento neoadyuvante

\begin{tabular}{|lcccc|}
\hline & pTONOMO & pT3NOMO & pT3N1MO & pT2N2MO \\
\hline cT3N0M0 (4) & 3 & 1 & & \\
cT2N2M0 (2) & 1 & & 1 & 1 \\
cT3N2M0 (2) & & 1 & & \\
cT4N0M0 (3) & 1 & & 1 & 1 \\
cT4N2M0 (3) & 2 & & 1 \\
cT2N3M0 (1) & & & \\
cT4N3M0 (1) & & & & \\
\hline
\end{tabular}

\footnotetext{
${ }^{1}$ Respuesta patológica completa.

$\mathrm{c}=$ estadio clínico.

$\mathrm{p}=$ estadio patológico.
} 
supervivencia libre de enfermedad fue de 14,26 meses (las medianas en ambos casos no se han alcanzado aún). La supervivencia el primer año fue del $73,3 \%$, y a los 2 años del 66,6\%. La supervivencia libre de enfermedad el primer año fue del $53,3 \%$, y a los 2 años permanecía igual. Se ha documentado recurrencia de la enfermedad en 7 de los 15 pacientes de cuyos datos se dispone hasta este momento, una de ellas local, 3 local y a distancia (2 óseas y una hepática) y 3 a distancia ( 2 en sistema nervioso central y una ósea). Así pues, en estos pacientes y con este esquema, el control local se estima en el $73,4 \%$, lo cual en tumores avanzados irresecables puede considerarse satisfactorio.

\section{Discusión}

El manejo del cáncer de pulmón no susceptible de tratamiento quirúrgico al diagnóstico ha sufrido importantes modificaciones en los últimos años, sin embargo poco se ha avanzado en cuanto a la supervivencia. El abordaje multidisciplinar de este tipo de tumores basado en diferentes combinaciones de quimioterapia, radioterapia y cirugía se ha convertido en una de las cuestiones más controvertidas y donde se realizan más ensayos en la actualidad. La incidencia de complicaciones postoperatorias tras una resección pulmonar por cáncer de pulmón se estima aproximadamente que es alrededor del $20 \%$, con una mortalidad en torno al $2 \%$ para una lobectomía y cerca del 5\% si se trata de una neumonectomía ${ }^{16,19,20}$. Una de las preguntas aún sin respuesta es si después de un tratamiento de inducción con radioterapia a dosis máximas es factible y seguro plantear la cirugía como tratamiento definitivo. En este sentido las series publicadas en la literatura arrojan resultados contradictorios. Fowler et $\mathrm{al}^{4} \mathrm{pu}-$ blican un estudio de 23 pacientes sometidos a 60 Gy de radioterapia con una mortalidad del $23 \%$, que aumenta al $43 \%$ si se practica una neumonectomía. Deutsch et $\mathrm{al}^{5}$ encuentran una mortalidad global del $33 \%$, y un aumento en la incidencia de fístulas del muñón bronquial tras radioterapia a dosis plenas. Dodoli et $\mathrm{al}^{7}$ publican una mortalidad del $9 \%$ tras tratamiento de inducción, $14 \%$ si se trata de una neumonectomía. Stamatis et $\mathrm{al}^{21}$ encuentran una mortalidad del $4,9 \%$ y una morbilidad del $44 \%$ tras cirugía en pacientes que han recibido quimioradio- terapia de inducción. En este estudio el índice de Karnofsky bajo y la edad avanzada fueron factores predictivos de mortalidad. En cuanto a morbilidad, además de los anteriores, resultaron ser factores predictivos las alteraciones ecocardiográficas y la no cobertura del muñón bronquial. Por el contrario otros trabajos encuentran una incidencia aceptable de complicaciones postoperatorias tras radioterapia $^{11-17}$. Recientemente, Sonnet et al $^{22}$ analizan 40 pacientes con CNMP localmente avanzado (EIII) que han recibido quimioterapia basada en cisplatino, y de manera concurrente dosis mayores de 59 Gy de radioterapia, y encuentran tan solo un caso de edema pulmonar postoperatorio y un caso de fístula del muñón bronquial. La supervivencia de estos pacientes al año, dos años y cinco años fue del 92,4\%, $66,7 \%$ y $46,2 \%$ respectivamente. La disparidad de estos resultados se puede explicar por la gran heterogeneidad de los diferentes estudios en cuanto al tamaño muestral, los criterios de selección, la clasificación de los pacientes (la realización de pruebas de diagnóstico invasivo) y las definiciones en cuanto a morbimortalidad perioperatoria, puesto que algunos estudios consideran como complicación postoperatoria tan sólo los eventos mayores, como infarto de miocardio, empiema, hemorragia o fístula del muñón bronquial; mientras que otros también recogen las complicaciones consideradas como menores tales como fuga aérea persistente, infección urinaria, atelectasias, etc.

Por otro lado, en nuestra serie, la elevada incidencia de neumonectomías izquierdas en el grupo de mayor dosis de radioterapia no se ha traducido en una mayor morbimortalidad postquirúrgica, seguramente debido a la selección cuidadosa de los pacientes candidatos a resección y a un óptimo manejo perioperatorio.

En nuestro trabajo se da una aparente paradoja, y es que se produce una mayor incidencia de complicaciones en valores absolutos en el grupo que recibe menores dosis de radioterapia, aunque las diferencias no resultan ser estadísticamente significativas. Dos posibles explicaciones a esta circunstancia son, en primer lugar, el pequeño tamaño muestral de ambos grupos, y en segundo lugar la confirmación de nuestra hipótesis de trabajo, que la morbilidad postoperatoria de los pacientes sometidos a radioterapia, pese a ser lógicamente mayor que en los pacientes que no se han sometido a ningún trata- 
miento de inducción, sea independiente de las dosis utilizadas. Esto vendría a desmentir los resultados de los primeros trabajos que anunciaban una morbimortalidad postquirúrgica inaceptable en los pacientes sometidos a tratamiento radioterápico preoperatorio, sobre todo para dosis mayores de $59 \mathrm{~Gy}^{4}$. Quizá en la década de los 90 esto fuera así, pero la realidad hoy en día es muy distinta, y es que la precisión alcanzada en la planificación tridimensional preoperatoria de la radioterapia, consiguiendo la mínima exposición a radiación de los tejidos adyacentes a la diana terapéutica ${ }^{12}$, añadido al perfeccionamiento de la técnica quirúrgica, cubriendo de rutina el muñón bronquial de estos pacientes cuando se hace una neumonectomía o tras una lobectomía en la que el muñón quede desprotegido (reduciendo así al máximo la dehiscencia de la sutura bronquial), así como la aplicación de medidas encaminadas a reducir la incidencia de insuficiencia respiratoria en las unidades de recuperación postquirúrgica (restricción hídrica, mínimo aporte de oxígeno, ventilación protectora y fisioterapia respiratoria intensiva con movilización precoz) han conseguido que se pueda intervenir a este tipo de pacientes con una morbimortalidad postoperatoria aceptable.

Los datos de los que disponemos hasta el momento en cuanto a supervivencia son realmente elevados, con una supervivencia a los dos años del $66 \%$, esto quizá pueda verse influido por el hecho de que incluimos en nuestra serie a un número elevado de pacientes con tumores de Pancoast T3N0M0, que de manera estándar reciben quimioterapia y radioterapia antes de la cirugía ${ }^{23}$ y que tienen mejor pronóstico que el grupo de pacientes con enfermedad localmente avanzada (estadio III)

Por último cabe reseñar las notables limitaciones de este trabajo, que deben hacernos ser prudentes en la interpretación de los resultados. Se trata del análisis retrospectivo en una muestra de pacientes muy concreta de una única institución, lo cual no permite extrapolar los resultados a otros grupos quirúrgicos. Además el tamaño muestral es escaso, y puede habernos impedido encontrar diferencias en la comparación entre grupos (error de tipo II). Sin embargo, a pesar de sus limitaciones estadísticas, este tipo de trabajo puede ser útil a la hora de analizar pacientes con las mismas características de otras series mediante estudios de metaanálisis.

\section{Conclusiones}

En nuestra opinión se necesitan estudios prospectivos multiinstitucionales que confirmen estos resultados. Hasta que se alcance ése nivel de evidencia nos parece razonable ofrecer tratamiento quirúrgico a los pacientes que tras alcanzar alguna respuesta con dosis plenas $(>59)$ de radioterapia \pm quimioterapia sigan siendo operables.

\author{
Correspondencia: \\ Dr. D. Gómez de Antonio \\ Servicio de Cirugía Torácica \\ Hospital Universitario Puerta de Hierro \\ San Martin de Porres, 4 \\ E-28035 Madrid \\ dgavm@yahoo.es
}

\section{Bibliografía}

1. Auberg AJ, Samet JM. Epidemiology of lung cancer. Chest 2003;123:21S-49S.

2. Martini N, Flettinger BJ. The role of surgery in N2 lung cancer. Surg Clin North Am 1987;67:1037-49.

3. Edelman MJ, Gandara DR, Roach M, Benfield JR. Multimodality therapy in stage III non small cell lung cancer. Ann Thorac Surg 1996;61:1564-72.

4. Fowler WC, Langer CJ, Curran WG, Keller SM. Postoperative complications after combined neoadyuvant treatment of lung cancer. Ann Thorac Surg 1993;55:986:9.

5. Deutsch M, Crawford J, Leopold K, Wolf W, Foster W, Herhdorn J et al. Phase II study of neoadyuvant chemotherapy and radiation therapy with thoracotomy in the treatment of clinically staged IIIA non-small cell lung cancer. Cancer 1994;74:1243-52.

6. Martin J, Ginsberg RJ, Abolhoda A, Rusch W, Bains MS, Downey RJ, Korst RJ, Weigel TL, Kris MJ. Morbidity and mortality after neoadyuvant therapy for lung cancer: the risks of right pneumonectomy.Ann Thorac Surg 2001;72:1149-54.

7. Doddoli C, Thomas $\mathrm{P}$, Thirion X, Serèe Y, Giudicelli R, Fuentes P. Postoperative complications in relation with induction therapy for lung cancer. Eur J Cardiothorac Surg 2001;20:385-90.

8. Bonomi P, Faber LP, Warren W, et al. Postoperative bronchopulmonary complications in stage III lung cancer patients treated with preoperative paclitaxel-containing chemotherapy and concurrent radiation. Semin Oncol 1997; 24:S12.

9. Macchiarini P, Chevalier AR, Monnet I, Vannetzel JM, Rebischung JL, Cerrina J, Parquin F, Le Roy Ladurie F, 


\section{Gómez de Antonio y cols.}

Lenot B, Dartevelle P. Extended operations after induction therapy for stage IIIB (T4) non-small cell lung cancer. Ann Thorac Surg 1994;57:966-973.

10. Semik M, Riesenbeck D, Linder A, et al. Preoperative chemotherapy with and without additional radiochemotherapy: benefit and risk for surgery of stage III non-small cell lung cancer. Eur J Cardiothorac Surg 2004;26:1205-10.

11. Sonnet JR, Krasna MJ, Suntharalingam M, Schuetz J, Doyle A, Lilenbaum R, et al. Safe pulmonary resection after chemotherapy and high-dose thoracic radiation. Ann Thorac Surg 1999;68:316-20-

12. Suntharalingam M, Sonnet JR, Haas ML, Doyle A, Schuetz J, et al. The use of concurrent chemotherapy with high dose radiation prior to surgical resection in patients presenting with apical sulcus tumor. Cancer J Sci Am 2000;6:365-71.

13. Mathisen DJ, Wain JC, Wright C, Choi N, Carey R, Hilgenberg A, Grossbard M, Linch T, Grillo H. Assesment of preoperative accelerated radiotherapy and chemotherapy in stage IIIA(N2) non-small-cell lung cancer. J Thorac Cardiovasc Surg 1996;111:123-33

14. Rusch VW, Albain KS, Crowley JJ, Rice TW, Lonchyna V, McKenna R, Livingston RB, Griffin BR, Benfield JR. Surgical resection of stage IIIa and stage IIIb non-smallcell lung cancer after concurrent induction chemoradiotherapy. J Thorac Cardiovasc Surg 1993;105:97-103.

15. Roth JA, Atkinson EN, Fosella F, et al. Long-term followup of parients enrolling in a randomized trial comparing perioperative chemotherapy and surgery with surgery alone in resectable stage IIIA non-small-cell lung cancer. Lung Cancer 1998;21:1-6.
16. Albain KS, Rusch VW, Crowley JJ, et al. Concurrent cisplatin/etoposide plus chest radiotherapy followed by surgery for stages IIIA (N2) and IIIB non-small-cell lung cancer: mature results of Southwest Oncology Group phase II study 8805. J Clin Oncol 1995;13:1880-92.

17. Sugarbaker DJ, Herndon J, Kohman LJ, Krasna MJ, Green MR. Results of cancer and leukemia group B protocol 8935. A multiinstitutional phase II trimodality trial for stage IIIA (N2) non-small-cell lung cancer. Cancer and Leukemia Group B Thoracic Surgery Group. J Thorac Cardiovasc Surg 1995;109:473-85.

18. Mountain CF: Revisions in the international system for staging lung cancer.Chest 1997;111:1710-7.

19. Deslauriers J, Ginsberg RJ, Piantadosi S, Fournier B. Prospective assessment of 30-day operative morbidity for surgical resections in lung cancer. Chest 1994;106:329S$30 \mathrm{~S}$.

20. Duque JL, Ramos G, Castrodeza J, et al. Early complications in surgical treatment of lung cancer; a prospective, multicenter study. Ann Thorac Surg 1997;63:944-50.

21. Stamatis G, Djuric D, Eberhardt WE, et al. Postoperative morbidity and mortality after induction chemoradiotherapy for locally advanced lung cancer. Eur J Cardiothorac Surg 2002;22:292-7.

22. Sonnet JR, Suntharalingam M, Edelman MJ, Patel AB, Gamliel Z, Doyle A, Hausner P, Krasna M. Pulmonary resection after curative intent radiotherapy (>59 Gy) and concurrent chemotherapy in non-small-cell lung cancer. Ann Thorac Surg 2004;78:1200-6.

23. Spira A, Ettinger DS. Multidisciplinary management of lung cancer. N Engl J Med 2004;350:379-92. 